\title{
Entre permanences et bifurcations : une lecture territoriale des destinations touristiques de montagne
}

Emmanuelle George-Marcelpoil, Coralie Achin, Gabriel Fablet et Hugues François

\section{(2) OpenEdition}

Journals

Édition électronique

URL : http://journals.openedition.org/tourisme/1237

DOI : $10.4000 /$ tourisme. 1237

ISSN : 2492-7503

Éditeur

Éditions touristiques européennes

Référence électronique

Emmanuelle George-Marcelpoil, Coralie Achin, Gabriel Fablet et Hugues François, « Entre permanences et bifurcations : une lecture territoriale des destinations touristiques de montagne », Mondes du Tourisme [En ligne], Hors-série | 2016, mis en ligne le 01 septembre 2016, consulté le 19 avril 2019. URL : http://journals.openedition.org/tourisme/1237 ; DOI : 10.4000/tourisme.1237

Ce document a été généré automatiquement le 19 avril 2019.

\section{c)}

Mondes du tourisme est mis à disposition selon les termes de la licence Creative Commons Attribution - Pas d'Utilisation Commerciale - Pas de Modification 4.0 International. 


\title{
Entre permanences et bifurcations : une lecture territoriale des destinations touristiques de montagne
}

\author{
Emmanuelle George-Marcelpoil, Coralie Achin, Gabriel Fablet et Hugues \\ François
}

\section{Introduction}

1 Les travaux menés depuis de nombreuses années à Irstea de Grenoble (ex-Cemagref) s'intéressent aux mutations des systèmes touristiques localisés (STL) (Perret, 1992) dans les espaces montagnards français, en questionnant les facteurs à l'œuvre dans ces dynamiques et le sens de leurs impacts. Partant des préoccupations opérationnelles des opérateurs publics et privés, parties prenantes de l'économie touristique, notre approche s'inscrit dans une démarche de recherche finalisée au service de l'action publique en matière de développement touristique des territoires. Pour ce faire, nous nous ancrons dans une posture de recherche valorisant des analyses empiriques fines de systèmes touristiques de montagne, en mobilisant essentiellement des informations qualitatives d'origines variées (entretiens semi-directifs, documents de planification, archives publiques) complétés par des données plus quantitatives et spatialisées (statistiques publiques, données métier, information géographique, matrice cadastrale). Dans cette contribution, nous nous proposons de revisiter nos travaux et recherches, en en précisant les corpus théoriques et analytiques mobilisés pour une lecture par essence pluridisciplinaire des systèmes touristiques de montagne, objets d'études privilégiés.

2 Dans une perspective d'aménagement du territoire, notre recherche s'intéresse aux mutations à l'œuvre dans les systèmes touristiques et plus particulièrement dans les stations de sports d'hiver. Elle donne une place centrale aux stratégies d'acteurs et à leurs modalités d'organisation pour le fonctionnement d'une activité cruciale pour le 
développement local. Ainsi, les stations se distinguent aujourd'hui par une multiplicité de trajectoires d'évolution, que nous proposons d'explorer et de qualifier dans leurs déterminants. Pour ce faire, nous reviendrons dans un premier temps sur la lecture des stations en économie et aménagement, en proposant de considérer ces dernières comme des coordinations d'acteurs localisées, et ce en filiation des travaux sur les districts industriels, les systèmes productifs localisés (SPL) et autres clusters. Cette analyse nous montrera une réalité contrastée, loin des stéréotypes traditionnellement admis opposant grande station d'altitude à station de moyenne montagne, se déclinant sur un continuum entre ces deux extrêmes.

Dans ces différentes déclinaisons, la place de l'acteur public fait l'objet d'une attention particulière, eu égard au statut spécifique des remontées mécaniques, service public sous la responsabilité des collectivités territoriales. Il joue donc un rôle déterminant, mais doit composer avec une variété de logiques et de stratégies dont la coordination et l'intégration structurent l'orientation et l'inflexion des trajectoires de développement. Le territoire dans lequel l'organisation des acteurs et de leurs ancrages prend corps nous intéresse particulièrement. Il s'agit de déterminer avec quelles intensités et sous quelles formes l'ancrage territorial est pris en compte par les différents opérateurs en présence, afin de mieux saisir les parts du générique et du spécifique des territoires de station.

Une seconde partie mettra donc l'accent sur les trajectoires des stations, dans leurs fondements fonctionnels comme spatiaux. Objet ancien dans la recherche en tourisme, l'analyse des processus de développement des stations touristiques constitue le cœur des travaux fondateurs de Plog (1974) et Butler (1980), prolongés par ceux de l'équipe Mit relatifs aux modalités de transformation des lieux touristiques (2005). En revisitant les théories de l'économie industrielle, les approches en termes de systèmes productifs (Perret, 1992) ont permis de dépasser le caractère normatif des approches typologiques en favorisant la prise en compte du milieu local dans les processus d'évolution des stations. Que ce soit à travers une analyse en termes d'ancrage (Marcelpoil, 2007a) ou encore le recours au concept de capital touristique (Darbellay et alii, 2011), le facteur territorial est une variable centrale pour comprendre le développement des stations. Les catégories classiques de l'analyse sont dès lors bousculées, mettant à mal l'opposition entre stations d'altitude et stations moyennes. Pour ces dernières, l'heure est à la diversification, fondée a priori sur la spécificité des ressources territoriales (François, 2007). Ceci questionne l'organisation du tourisme, avec une évolution du système d'acteurs impliqués et souvent un changement d'échelle. Dans ce cadre, le territoire et ses ressources permettent à la filière, au tissu productif de s'exprimer. Pour les stations d'altitude, souvent qualifiées d'archipels d'altitude (Wozniak, 2006, repris depuis par la Facim), la réalité est toute autre avec une filière productive qui continue de primer sur le territoire. Le caractère mondialisé des stratégies d'opérateurs (remontées mécaniques ou hébergement) constitue ainsi un facteur essentiel de l'explicitation des trajectoires de ces stations. Ces deux secteurs d'activité jouent en effet un rôle central dans le devenir d'une destination touristique. En ce sens, l'étude urbaine des stations constitue un révélateur des réalités structurelles et conjoncturelles des trajectoires des stations de montagne qui feront l'objet du dernier point de notre présentation. 


\section{Le jeu des acteurs au cœur de la gouvernance des systèmes touristiques localisés}

5 Un premier axe d'analyse concerne la manière dont le système touristique de montagne de type station peut être lu. Alors qu'elles ont longtemps été réduites à une logique de filière, nous avons, au début des années 2000, investi la thématique de l'organisation touristique en partant de l'hypothèse que le devenir des stations, leur capacité d'adaptation ou leur résistance face aux incertitudes de tous ordres sont largement dépendants de leur organisation, de leur pilotage. Comprendre les modalités de leur gouvernance, leurs points positifs comme leurs éventuels dysfonctionnements, permet de cerner, d'évaluer leurs marges de manœuvre pour l'avenir. Ce positionnement scientifique s'inscrit dans une action publique renouvelée, marquée par le retrait de l'État et l'affirmation corrélative des territoires. Elle réserve effectivement une place plus importante aux démarches collaboratives, contribue à l'émergence de nouveaux acteurs, et à de nouvelles formes d'articulation entre intérêts privés ou publics.

\section{La station : un système d'acteurs complexe}

6 Cette idée de gouvernance trouve ses racines au niveau international, mais surtout au niveau européen, avec l'expansion urbaine toujours plus nette dans les années 1980. Les principales métropoles européennes, qualifiées de "villes qui comptent», de "métropoles qui gagnent", ont à gérer des services publics d'envergure, tels les transports, la ressource en eau, etc. La prise en charge de tels équipements et services est alors fréquemment confiée à des entreprises privées, avec des phases de négociation intense, débouchant sur la définition de contrats de délégation entre d'un côté les acteurs publics, les collectivités, et de l'autre les acteurs privés, les entreprises offrant leurs services. C'est la reconnaissance du partenariat public-privé, qualifié de gouvernance urbaine, que le politologue Patrick Le Galès (1995) définit comme des arrangements formels et informels entre des intérêts privés comme publics. De tels arrangements sont dès lors cruciaux pour appréhender les décisions concrètement mises en œuvre, et in fine le sens donné aux dynamiques. Ce cadre analytique contribue également à revoir la frontière entre univers public et privé et la définition d'un intérêt commun, support des stratégies d'acteurs.

7 L'application de la notion de gouvernance aux stations dans les Alpes comme dans d'autres contextes territoriaux (Gerbaux et Marcelpoil, 2006 ; Marcelpoil, 2008) a montré la complexité du système d'acteurs, loin du seul face-à-face entre collectivités locales et opérateurs de remontées mécaniques, qui fut au centre de la création et de l'expansion des stations. Ainsi, si la loi Montagne ${ }^{1}$ a reconnu la place des collectivités locales supports, les analyses empiriques soulignent à l'envi la diversité des acteurs, des logiques et surtout la difficulté à définir et porter un référentiel commun pour la station et son territoire. Dans ce contexte, les stations peuvent être appréhendées comme des coordinations d'acteurs, fruits d'une histoire longue et mobilisant, aux côtés de l'économie, des facteurs culturels ou politiques spécifiques. 


\section{L'enjeu du pilotage des destinations}

8 En pratique, aux côtés de l'opérateur de remontées mécaniques, dont le statut est varié (Marcelpoil et François, 2012), la sphère des acteurs économiques, parties prenantes de la destination, est très composite. Par exemple, dans le domaine de l'hébergement, des structures dépendantes de groupes nationaux ou internationaux côtoient des prestataires indépendants. Pourtant, bien que de statuts, de tailles et d'implications divers dans les stations, ces acteurs participent tous de la destination, de son fonctionnement et in fine de son devenir. C'est bien tout l'enjeu ces dernières années des démarches de gouvernance : faire adhérer la diversité des acteurs économiques à un projet de destination et concilier leur logique économique individuelle à l'intérêt collectif de la station.

9 Cette notion d'implication dans un système d'acteurs composite pour le bien commun que représente la station concerne également la sphère civile. Cette dernière, souvent occultée dans le fonctionnement de la station, contribue pourtant au financement de la collectivité locale, ne serait-ce que par le biais des impôts. Dans ce contexte, plusieurs sites ont vécu l'émergence de revendications de certaines catégories de populations, résidant ou travaillant en station. Ce sont par exemple les actifs saisonniers, maind'œuvre indispensable pour le fonctionnement de la station mais dont le pouvoir d'achat est entravé par des difficultés d'accès aux services, au logement et encore plus au foncier. Ce constat de formes de précarisation, à différencier selon les parcours individuels des saisonniers (Fatiga, 2010), a poussé les pouvoirs publics et les partenaires socioéconomiques à élaborer des réponses adaptées, dont les maisons des saisonniers constituent un bel exemple. La sphère civile se structure également autour des résidents secondaires, dont la contribution en stations a été pointée du doigt, souvent sévèrement, via le phénomène des volets clos, handicapant la qualité du remplissage de la station. Ces résidents secondaires ont alors fait entendre leur voix, et leur contribution est souvent ancrée historiquement, dans la genèse comme dans le développement de la station. In fine , ces quelques illustrations mettent en exergue la difficulté de pilotage pour la collectivité locale support, certes reconnue comme pilote touristique mais qui doit faire face, avec des compétences et des budgets pas toujours adaptés, à un système d'acteurs complexe et difficile à mener vers la constitution d'un acteur collectif.

10 Sur un plan plus analytique, l'analyse fine menée sur un panel de stations souligne la diversité des relations comme de leurs dynamiques, l'influence des facteurs historiques, culturels et sociaux dans l'évolution de la station. Un constat qui nous a conduit, dans la foulée des travaux portant sur les districts industriels italiens (Beccatini, 1992) et les systèmes productifs localisés en France (Courlet et Pecqueur, 1992) et autres clusters (Porter, 1985), à concevoir les stations de sports d'hiver comme des systèmes touristiques localisés (STL) (Perret, 1992).

\section{La diversité des trajectoires d'évolution des systèmes touristiques}

11 Partant de ce corpus théorique des STL de montagne, l'analyse de leurs trajectoires a questionné les logiques des acteurs parties prenantes de ces systèmes. En particulier, l'objectif a consisté à cerner la part du territoire dans les stratégies des opérateurs, gestionnaires de remontées mécaniques et d'hébergement, et in fine l'évaluation des 
modalités d'ancrage de ces destinations (Marcelpoil, 2007a). Cette question de l'ancrage interroge également la dynamique des stations : tout d'abord celle des stations moyennes, au travers de l'injonction d'une diversification touristique assumée, et valorisant la spécificité de leurs ressources territoriales. Celle des stations d'altitude, ensuite, souvent perçues comme des «archipels d'altitude» mais dont le fonctionnement souligne la réalité des relations entretenues avec leur territoire support.

\section{Les stations de moyenne montagne en quête d'un idéal renouvelé}

12 Les sites aujourd'hui qualifiés de "stations de moyenne montagne» ont connu depuis l'après-guerre un développement dans le sillage de celui des stations d'altitude issues du Plan neige, sans toutefois prétendre, par leurs caractéristiques (altitude, pente, orientation des pistes...), à la même intensité de développement. En pratique, les aléas conjoncturels survenus depuis la fin de la décennie 1990 ont eu une résonnance particulière auprès de ces stations de moyenne montagne, allant jusqu'à questionner l'origine conjoncturelle ou structurelle des difficultés rencontrées (ANEM, 1991), voire la pertinence de leur modèle de développement (CNM, 2010)

13 Loin de rester étrangers à cette sévère désillusion autour des bénéfices acquis de l'« or blanc» (Mouriquand, 1988) en moyenne montagne, les pouvoirs publics se sont rapidement mobilisés afin de mettre en place un accompagnement public dédié à maintenir la vocation originelle d'aménagement du territoire des stations concernées (Perret, 1992). Ainsi, prenant le pas de la politique nationale transcrite au travers des plans pluriannuels de développement touristique institués par la directive de 1977, l'accompagnement aux stations sera à compter de cette période mis en œuvre à un niveau inférieur par les collectivités locales des différents niveaux. Ce sont alors dans un premier temps la professionnalisation des acteurs et l'atténuation des impacts de ces variations conjoncturelles, en particulier par le financement d'équipements de production de neige de culture ou encore l'accompagnement à la restructuration des domaines skiables, qui ont constitué le cœur des premières générations de politiques publiques. La reconduction de ces politiques a toutefois entraîné un renouvellement des actions éligibles. Désormais, la diversification de l'offre touristique des stations tend à devenir un leitmotiv prégnant dans l'ensemble des contextes territoriaux ${ }^{2}$.

Des bouleversements d'ampleur sont ainsi en cours dans les territoires concernés : alors que les sites de moyenne montagne s'étaient structurés autour d'un produit générique, la mise en œuvre progressive de la diversification des activités touristiques impose quant à elle la valorisation des différenciations et spécificités territoriales (Escadafal, 2004). Ce sont dès lors les différentes ressources locales, qu'elles soient sociales ou culturelles (Dissart, 2012), qui seront valorisées en complément de l'activité touristique traditionnelle. Ainsi, la mobilisation nouvelle de telles ressources, conjuguée au développement des migrations d'agrément dans les territoires montagnards périurbains, se traduisant parfois par des conflits d'usage entre la fonction touristique originelle et la nouvelle fonction résidentielle, nous conduit à modifier le regard jusqu'alors porté aux stations. L'appréhension du développement touristique à une échelle territoriale suprastation, où cette dernière n'est plus perçue comme une entité indépendante, devient alors indispensable. Elle contribue notamment à diluer le poids structurant de l'acteur remontées mécaniques, à l'origine constitutif d'une station comme destination touristique dédiée aux sports d'hiver. En définitive, ce sont tout autant les questions du 
redimensionnement du territoire touristique, intégrant de facto la pluralité des ressources mobilisées en vue de l'élargissement de l'offre touristique, que celles de l'implication et de la coordination des acteurs, qui concentrent les débats.

Institué à travers la notion de territoire de projet, le déport du regard de l'échelle station à l'échelle supra-station suppose la définition d'un territoire touristique "pertinent et cohérent " en vue de la réalisation d'actions de diversification. Encouragé localement dans le cadre de la territorialisation des politiques publiques (Epstein, 2004) via l'incitation à la constitution d'espaces valléens (Conseil régional Provence-Alpes-Côte d'Azur, 2006) ou encore de pôles touristiques (Comité de massif des Pyrénées, 2007), ce redimensionnement des territoires vient bouleverser leur organisation traditionnelle. Au-delà, cette question du périmètre de l'action publique touristique locale soulève bien d'autres interrogations. En premier lieu, c'est ainsi la question de la structure administrative porteuse du projet touristique, et par là-même l'existence d'une cohérence entre le territoire administratif et le territoire de projet qui est posée. En effet, alors que "l'identification de la destination par un touriste ne correspond pas forcément à un territoire au sens administratif » (Escadafal, 2004), l'existence d'un porteur unique pour ces projets de territoire conditionne bien souvent sa recevabilité. Ainsi, tandis que l'échelon intercommunal est bien souvent porté au cœur du projet touristique (Vlès, 2001), d'autres modalités de coopération locale coexistent par le biais de structures administratives alternatives, à l'image des syndicats mixtes des parcs naturels régionaux et autres syndicats de communes (communauté de communes, syndicats mixtes, Sivu ou Sivom) qui s'imposent parfois comme les relais d'une politique touristique. Au-delà de l'identification de la structure administrative ad hoc se pose également la question de ses compétences. Dans un contexte où la compétence tourisme ne fait en effet que très rarement l'objet d'une délégation aux contours clairs (limites des formes de délégation partielles?) au profit d'une échelle supra-communale, quelles peuvent en être les conséquences sur la définition et la mise en œuvre d'un territoire de projet et d'un projet pour le territoire?

Considérée comme étant le premier pilier de la construction territoriale (Hadjou, 2009), la coordination des acteurs constitue également une question fondamentale nécessitant d'être intégrée au cœur des réflexions. Appréhendés au travers de la notion de gouvernance, les systèmes d'acteurs composant la station de montagne se sont complexifiés avec la mise en œuvre de la diversification. C'est désormais une large palette d'acteurs qui sont peu à peu intégrés aux réflexions, devant dorénavant être partagées, imposant de porter une attention toute particulière à ces systèmes en évolution.

17 Ainsi, à travers des questionnements en termes de jeux d'échelle, de structures administratives compétentes mais également d'acteurs impliqués, c'est bien ici la question de l'organisation territoriale des territoires supports de stations de moyenne montagne qui est posée.

\section{L'ancrage territorial à degré variable des stations d'altitude}

18 À plus haute altitude, les stations sont plus directement les héritières de la doctrine d'aménagement portée par les services de l'État d'après-guerre. Fort de son rôle dans la politique de reconstruction, l'administration de l'Équipement va prendre une place centrale dans l'aménagement touristique de la montagne, notamment au travers de l'aventure de Courchevel (François et Marcelpoil, 2012). L'invention d'une nouvelle forme 
d'urbanisation dédiée aux loisirs, dans un territoire qui n'a encore fait l'objet que de peu d'attention de la part du pouvoir central, impulse une dynamique d'innovation qui s'affranchit facilement des cadres préexistants. Faute de moyens d'action adaptés, la finalité prime sur toute chose et le concept de station s'impose, avec la prééminence d'une conception fonctionnelle de l'organisation de l'espace pour la pratique du ski et du tourisme. Ainsi, si l'intégration au milieu montagnard n'est pas absente des préoccupations, elle s'affirme avant tout dans une mise en scène de la nature par la station (Chevallier, 1996), faisant peu de cas d'autres caractéristiques territoriales. Cette mise à l'écart des populations locales et des usages préexistants, déjà en germe à Courchevel (François et Marcelpoil, 2012) se renforce avec la diffusion du modèle de la station intégrée également désignée comme station « en site vierge » ou « ex nihilo». La constitution d'une réserve foncière préalable a reposé sur l'exclusion des populations locales avec la pratique systématique de l'expropriation au profit d'un "promoteur unique ». La systématisation de la méthode renforce son caractère implacable, durcissant les oppositions locales, comme elle a pu s'exprimer avec violence dans le cas des Arcs. Généralement considérées comme de véritables paquebots des neiges, ces stations de troisième génération renvoient ainsi l'image d'une politique le plus souvent qualifiée d'aterritoriale.

Une étude fine de leurs modalités d'implantation montre au contraire un réel ancrage dans la réalité territoriale, avec la constitution d'un véritable milieu innovateur (Marcelpoil et alii, 2010) sur fond de changement social. Dès l'origine, l'expérience de Courchevel repose sur l'impulsion donnée par le département de la Savoie à la recherche de nouvelles voies de développement économique. Précocement, elle a impliqué des acteurs d'origine locale et son aura a contribué à faire de la Savoie un pôle attractif de l'aménagement touristique de la montagne. Aux Arcs, Roger Godino, promoteur unique incarnant la vision de la "station intégrale » inspirée par le succès du Club Méditerranée, a lui-même des racines savoyardes. Nombre de ces pionniers du développement montagnard partagent un parcours de formation qui les a amenés en dehors de leur territoire d'origine mais pour lequel ils conservent un fort attachement. En s'appuyant sur ces relais techniques de la modernité en zone de montagne, les acteurs politiques de la problématique montagnarde vont lui donner une envergure nationale qui contribue directement au changement social «par le haut» (François et George-Marcelpoil, 2015). Ceci explique une rotation des élites qui conduit à l'adaptation des économies montagnardes au régime capitaliste, se substituant alors à celui des économies paysannes (Préau, 1983 et 1984). Afin de conduire cette transition vers la "modernité », le promoteur unique joue un rôle aussi symbolique que concret. Du point de vue de l'image, il incarne le capitaine de navire possédant l'ensemble des moyens nécessaires pour accompagner les territoires de stations vers les nouvelles réalités économiques. Garante de l'unité des différentes composantes d'une station, l'action du promoteur unique a participé de manière plus directe à leur intégration fonctionnelle et à la cohérence de l'offre touristique associée.

20 Néanmoins, si son rôle a trouvé toute sa légitimité dans la phase de construction des stations jusqu'au milieu des années 1980, la phase de gestion qui a succédé a remis en cause sa légitimité. L'affirmation d'intérêts multiples en station de montagne marque effectivement la fin de la prédominance du principe de promoteur unique. L'absence d'un responsable clairement désigné laisse une place croissante à la variable territoriale telle que le consacrent les articles 42 et 46 de la loi Montagne, conférant un rôle clef aux 
collectivités locales. Il est d'ailleurs remarquable de constater que l'affirmation de la problématique de la gouvernance (Gerbaux et alii, 2004 ; et voir également la première partie de notre article) s'est souvent traduite par la recherche de l'organisation locale la plus à même de réunir les acteurs et d'appréhender les questions relatives aux stations dans leur globalité. Cependant, cette part de la réalité ne recouvre pas l'ensemble des facteurs déterminant les dynamiques actuelles de ces sites d'altitude. Pour cela, il est indispensable de prendre en considération l'organisation des acteurs polarisant l'offre touristique en station.

21 L'approche intégrée du développement cède le pas à une approche par filière privilégiant la maximisation sectorielle du volume d'activité et des profits. Dans ce sens, la structuration des acteurs privés fait contrepoids à la régulation par les collectivités locales pour imprimer leur propre rythme au développement des stations (Marcelpoil et François, 2012). Le département savoyard, à l'origine moteur de l'innovation, a su réinventer ainsi les modalités de son intervention avec la constitution de Savoie Stations Participations. Inspirée du modèle des holdings privées, cette structure présente l'originalité de compléter l'objectif de rentabilité avec ceux de l'aménagement du territoire et de péréquation. La structuration des acteurs parties prenantes des stations engage essentiellement ceux de la filière remontées mécaniques, offre motrice pour l'ensemble de l'économie de la station. Néanmoins, moteur n'est pas synonyme d'indépendance, et leur propre fonctionnement ne peut faire l'économie de variables locales, notamment celles liées au séjour des touristes dans la station. Hérités de la phase originelle de construction, les phénomènes fonciers et immobiliers apparaissent donc comme une clef de lecture fertile des dynamiques territoriales à l'œuvre dans les stations, dont les conséquences sur le plan économique peuvent orienter le développement de ces dernières vers des trajectoires distinctes et parfois originales.

\section{L'impact du modèle de développement sur les dynamiques foncières et immobilières}

L'hétérogénéité des processus d'émergence des stations du massif alpin a en effet donné naissance à une grande diversité de configurations morphologiques (Cumin, 1970). Qu'elles soient héritées d'un modèle de développement "spontané " ou encore " planifié » (Dewailly et Flament, 1993), le contexte d'émergence tout comme le modèle urbain dans lequel ces stations ont puisé leur inspiration ne sont pas totalement étrangers dans la nature des dynamiques de croissance immobilière (Fablet et Marcelpoil, 2013). Ces paramètres déterminent en partie leur positionnement dans la hiérarchie urbaine mais participent aussi activement au caractère singulier des structures urbaines émergentes, lesquelles dépassent désormais le périmètre communément admis de la station comme unité touristique fonctionnelle.

23 Tandis que les stations d'altitude contemporaines du «Plan neige » ont fait l'objet d'une implantation volontairement située « à l'abri des clientèles de week-end ( (Lavaud, 1970), il n'en est pas de même pour un certain nombre de stations de moyenne montagne confrontées au phénomène périurbain. Le processus de rerésidentialisation que connaissent certaines de ces stations peut se traduire parfois par une conversion des hébergements touristiques en résidences principales, à même d'engendrer des mutations significatives du modèle économique sur lequel elles reposent (François, 2007 ; Marcelpoil 
, 2007b). Mises à l'écart des phénomènes résidentiels et basées sur les besoins d'une économie touristique hautement capitalistique, les stations d'altitude connaissent elles des phénomènes alternatifs. Marquées par les lourds héritages d'un modèle urbain fonctionnaliste et fragilisées par l'incapacité d'identifier les leviers nécessaires pour répondre à l'enjeu colossal de la réhabilitation (Miquel et alii, 2010), la construction de lits neufs apparait comme l'unique remède à l'érosion progressive de leur parc d'hébergement marchand.

\section{Le rôle de la construction dans l'évolution des stations} construction pour assurer sa viabilité (Fablet, 2013). Systématiquement invoqué pour justifier les opérations sur le plan règlementaire, le phénomène des "lits froids » ne constitue qu'un facteur visible de la réalité des ressorts économiques et institutionnels qui animent une telle frénésie de construction.

\section{Quelle autonomie du pouvoir local face à la globalisation ?}

Les dynamiques spéculatives autour de l'immobilier en stations ne sont pas uniquement le fait d'impératifs de fréquentation touristique. Elles répondent également à des modalités spécifiques de gouvernance urbaine dans lesquelles interagissent de larges réseaux d'acteurs, dont le modèle économique est étroitement lié à cette dynamique de croissance. Si la mise en marché régulière d'hébergements neufs permet aux exploitants le maintien de leurs objectifs financiers, elle constitue également une aubaine pour quelques promoteurs spécialisés qui ont trouvé, avec les dispositifs de défiscalisation, un 
marché de niche propice à la réalisation de marges confortables. Pour les communes, la commercialisation de quelques hectares de terrain communal est souvent perçue comme une opportunité économique pour permettre le financement d'équipements publics coûteux.

De l'élu au promoteur en passant par l'opérateur de remontée mécanique et le propriétaire foncier, ce sont bien des formes de coalitions de croissance (Logan et Molotch, 1987) qui s'opèrent à l'image d'autres destinations hivernales (Gill, 2000; Clifford, 2002 ; Clivaz et Nahrath, 2010). Les stations françaises les plus capitalistiques s'apparenteraient ainsi à de véritables growth machine (Molotch, 1976), où derrière l'impératif d'aménagement du territoire se déploie une logique mêlant étroitement les impératifs de la croissance économique avec ceux d'une maximisation de la rente foncière. En définitive, l'accroissement des marges de manœuvres lié au renouvellement des modalités d'exercice du pouvoir local en matière de planification foncière et touristique (Duboeuf, 2006) ne contraint guère le déploiement des logiques financières d'un marché globalisé. Au contraire, il semblerait que ce changement de paradigme dans les modalités de construction de l'action publique locale facilite les phénomènes de croissance immobilière.

La dialectique entre le local et le global dans les modalités de production urbaine en stations renouvelle et complexifie les formes d'ancrage, avec un fort impact sur leurs trajectoires de développement. L'approche par les phénomènes urbains est donc un outil particulièrement adapté pour décrire le rôle du facteur territorial dans ces processus. L'étude des discours de légitimation et leur formulation dans les stratégies locales de planification montrent toute la diversité des chemins empruntés par les systèmes touristiques et leur degré de dépendance au sentier (Piore et Sabel, 1984 ; Pierson, 1993). Néanmoins, l'analyse soulève les limites de l'argumentaire justifiant les opérations immobilières pour comprendre les ressorts de la croissance urbaine des stations. Face à un contexte règlementaire qui se veut contraignant pour l'urbanisation en montagne (Juen, 2012), l'enjeu majeur des recherches relatives aux dynamiques de production urbaine dans ces espaces se situe précisément dans la nécessité de s'affranchir du discours de légitimation pour questionner le véritable degré d'autonomie du local dans les dynamiques de développement.

\section{Conclusion}

En conclusion, l'analyse des trajectoires d'évolution des stations, prises comme des coordinations localisées d'acteurs, en souligne toute la richesse mais aussi toute la complexité. L'histoire de l'aménagement touristique de la montagne s'est traduite par une variété des arrangements entre des acteurs centrés sur l'objectif de création d'un outil de production à finalité touristique. La phase de gestion actuelle, trouvant son origine dans les premières difficultés de l'économie des sports d'hiver des années 1980, a de son côté mis en relief les logiques différenciées des acteurs et l'exacerbation de certains points de frottements au sein du système d'acteurs. Ainsi, la concentration du secteur des remontées mécaniques a modifié les relations de pouvoir avec les autorités organisatrices, lesquelles ont à définir et porter une stratégie territoriale partagée. Dans ce cadre, la place du foncier mais aussi de l'immobilier est centrale dans le devenir de la destination. C'est également tout le poids du changement global, dont la déclinaison climatique s'avère structurante aujourd'hui pour la pérennité du modèle économique de 
ces outils de production que sont les stations comme du modèle d'aménagement plus global. En la matière, les perspectives sont larges, mettant en avant le renforcement de l'économie des sports d'hiver pour les stations d'altitude ou invoquant la nécessité de la diversification pour les sites localisés en moyennes montagnes. In fine, l'objectif est de cerner les marges de manœuvres des collectivités locales, en relation avec les choix propres aux opérateurs économiques touristiques. C'est, sous-jacente, toute la richesse de l'identification et de l'explicitation des trajectoires d'évolution des stations, qui donneront à voir une gamme beaucoup plus large que les seules catégories des stations moyennes comme des stations d'altitude.

31 La caractérisation de ces trajectoires, qui oscillent entre permanences et bifurcations, entend nourrir une réflexion de l'action publique, bien entendu celle des acteurs porteurs de politiques publiques mais également celle des opérateurs économiques. En effet, bien que ces derniers puissent, a priori, sembler moins sensibles à ces recherches qui ne contribuent pas directement et immédiatement à la mise en œuvre de leur modèle économique, ils n'en demeurent pas moins en demande de réflexion prospective à moyen et long termes. L'identification des facteurs d'inflexion comme de rigidité des trajectoires de développement s'avère pertinente pour anticiper le devenir des territoires porteurs de stations. En pratique, ces dernières doivent composer avec le futur climatique dont les incertitudes masquent bien souvent la menace, affectant ainsi la mise en œuvre de stratégies d'adaptation pourtant indispensables.

Dans ce cadre, la poursuite d'analyses empiriques fines s'avère indispensable. Elle doit également s'assortir d'un effort de montée en généralité, qui résume et peut être assèche la richesse du terrain mais est garante d'une diffusion et d'une mise en perspective dans un cadre européen et international. En ce sens, notre récente démarche de conception d'un système d'informations sur l'ensemble des stations du massif des Alpes pour le compte de la Datar Alpes s'inscrit précisément dans ces objectifs analytiques.

\section{BIBLIOGRAPHIE}

Association nationale des élus de la montagne (ANEM), Stations de sports d'hiver, difficultés conjoncturelles ou structurelles ?, congrès d'Albertville, 1991.

Giacomo Becattini, « Le district industriel : milieu créatif », Espaces et Sociétés, nº 66, 1992, p. $147-164$.

Richard W. Butler, « The concept of tourist area cycle of evolution: implications for management of resources ", Canadian Geographer, vol. 24, n 1, 1980, p. 5-12.

Marc Chevallier, « Paroles de modernité. Pour une relecture culturelle de la station de sports d'hiver ", Revue de géographie alpine, vol. 84, n³ 1996, p. 29-39 [http://www.persee.fr/doc/ rga_0035-1121_1996_num_84_3_3868].

Hal Clifford, Downhill Slide: Why the Corporate Ski Industry is Bad for Skiing, Ski Towns, and the Environment, Sierra Club Books, 2002. 
Christophe Clivaz et Stéphane Nahrath, « Le retour de la question foncière dans l'aménagement des stations touristiques alpines en Suisse », Revue de géographie alpine, n 98-2, 2010 [https:// rga.revues.org/1220].

Conseil national de la montagne (CNM), Devenir des stations de moyenne montagne. Rapport du groupe de travail $n^{\circ} 1,2010$.

Comité de massif des Pyrénées, Convention interrégionale de massif des Pyrénées 2007-2013, 2007.

Conseil régional Provence-Alpes-Côte d'Azur, Programme d'aménagement durable et solidaire des stations des Alpes du Sud (PADS), 2006-2012, 2006.

Claude Courlet et Bernard Pecqueur, « Les systèmes industrialisés en France : un nouveau modèle de développement ", dans Georges Benko et Alain Lipietz (dir.), Les Régions qui gagnent. Districts et réseaux: les nouveaux paradigmes de la géographie économique, Presses universitaires de France, 1992, p. 81-102.

Georges Cumin, « Les stations intégrées », Urbanisme, nº 116, 1970, p. 50-53.

Frédéric Darbellay, Christophe Clivaz, Stéphane Nahrath et Mathis Stock, « Approche interdisciplinaire du développement des stations touristiques. Le capital touristique comme concept opératoire ", Mondes du tourisme, $\mathrm{n}^{\circ}$ 4, 2011, p. 36-48.

Jean-Michel Dewailly et Émile Flament, Géographie du tourisme et des loisirs, Sedes, 1993. Jean-Christophe Dissart, « Co-construction des capacités et des ressources territoriales dans les territoires touristiques de montagne : étude de cas sur l'Oisans ", Revue de géographie alpine, $\mathrm{n}^{\circ}$ 100-2, 2012 [https://rga.revues.org/1781].

Domaines skiables de France, Indicateurs et analyses 2012, 2012 [http://www.domaines-skiables.fr/ $\mathrm{fr} /$ publications/observatoire/]

Thomas Duboeuf, « Pouvoir local et stratégies foncières en stations de montagne françaises : quelle durabilité du développement touristique local et quels enjeux pour la gouvernance ? », Revue de géographie alpine, vol. 94, $\mathrm{n}^{\circ}$ 1, 2006, p. 33-41 [http://www.persee.fr/doc/ rga_0035-1121_2006_num_94_1_2382].

Renaud Epstein, « Après la territorialisation, la différenciation territoriale », Pouvoirs locaux, $\mathrm{n}^{\circ}$ 63, 2004, p. 35-42.

Alain Escadafal, « Aménagement touristique : quelles ressources territoriales au service de l'attractivité des destinations?", Montagnes méditerranéennes, $n^{\circ}$ 20, 2004, p. 97-102.

Gabriel Fablet, « La croissance immobilière des stations de sports d'hiver en Tarentaise. Entre vulnérabilités conjoncturelles et dérèglements structurels ", Revue de géographie alpine, $\mathrm{n}^{\circ}$ 101-3, 2013 [https://rga.revues.org/2188].

Gabriel Fablet et Emmanuelle Marcelpoil, « Formes et impacts des dynamiques foncières et immobilières en stations de montagne : l'exemple du massif alpin », dans Jean-François Joye (dir.), L'urbanisation en montagne. Observations depuis le versant juridique, Université de Savoie, 2013, p. 43-59.

Antoine Fatiga, «La dimension sociale dans les stations : un point de vue syndical », dans Emmanuelle George-Marcelpoil, Liliane Perrin-Bensahel et Hugues François (dir.), Les Stations de sports d'hiver face au développement durable. État des lieux et perspectives, L'Harmattan, 2010, p. 105-110. 
Hugues François, De la station ressource pour le territoire au territoire ressource pour la station : le cas des stations de moyenne montagne périurbaines de Grenoble, thèse de doctorat en aménagement de l'espace, université Joseph-Fourrier de Grenoble, 2007.

Hugues François et Emmanuelle Marcelpoil, « Vallée de la Tarentaise : de l'invention du Plan neige à la constitution d'un milieu innovateur dans le domaine du tourisme d'hiver ", Histoire des Alpes, $\mathrm{n}^{\circ}$ 17, 2012, p. 227-242.

Hugues François et Emmanuelle George-Marcelpoil, « Les racines locales du Plan neige : le changement social en Savoie ", dans Véronique Antomarchi (dir.), Tourisme de neige et de glace dans les régions septentrionales. Réalités et enjeux, L’âge d'homme, 2015, p. 163-176.

Françoise Gerbaux, Vincent Boudières et Emmanuelle Marcelpoil, « De l'utilité de la notion de gouvernance pour analyser les modes de management touristique locaux : l'exemple de la station des Arcs ", Ingénieries, $n^{\circ}$ 37, 2004, p. 75-85.

Françoise Gerbaux et Emmanuelle Marcelpoil, « Gouvernance des stations de montagne en France : les spécificités du partenariat public-privé », Revue de géographie alpine, vol. 94, nº 1, 2006, p. 9-19.

Alison Gill, « From growth machine to growth management: the dynamics of resort development in Whistler, British Columbia », Environment and Planning A, vol. 32, n 6, 2000, p. 1083-1103.

Lamara Hadjou, « Les deux piliers de la construction territoriale: coordination des acteurs et ressources territoriales », Développement durable et territoires. Économie, géographie, politique, droit, sociologie, Varia (2004-2010), 2009 [http://developpementdurable.revues.org/8208].

Philippe Juen, « L'érosion des principes d'urbanisation issus de la loi Montagne », Construction Urbanisme, $\mathrm{n}^{\circ}$ 11, 2012.

Bernard Lavaud, Les Sports d'hiver en France, La Documentation française, 1970.

Patrick Le Galès, « Du gouvernement des villes à la gouvernance urbaine », Revue française de science politique, vol. 45, $\mathrm{n}^{\circ}$ 1, 1995, p. 57-95 [http://www.persee.fr/doc/

rfsp_0035-2950_1995_num_45_1_403502].

John R. Logan et Harvey L. Molotch, Urban Fortunes: the Political Economy of Place, University of California Press, 1987.

Christian Mantei (dir.), Frédéric Berlioz et Guy Faur, Les Chiffres clés du tourisme de montagne en France, Atout France, 2009 (7éd.).

Emmanuelle Marcelpoil, «L'ancrage territorial des stations de montagne : quelles trajectoires et marges de manœuvre? ", dans Philippe Bourdeau (dir.), Les Sports d'hiver en mutation : crise ou révolution géoculturelle?, Hermes Science Publications, 2007a, p. 161-172.

Emmanuelle Marcelpoil (dir.), Nouvelles Pratiques touristiques en zones de montagne : vers un renouvellement des pratiques de gestion foncière?, Direction du Tourisme, $2007 \mathrm{~b}$.

Emmanuelle Marcelpoil, Les Trajectoires d'évolution des destinations touristiques de montagne, thèse d'habilitation à diriger des recherches, université de Pau et des pays de l'Adour, 2008.

Emmanuelle Marcelpoil, Hugues François et Sylvain Billet, L'Ancrage du financement des stations de la vallée de la Tarentaise : une lecture territoriale, Rapport pour le Conseil général de la Savoie, 2010. Emmanuelle Marcelpoil (dir.), Hugues François, Atlas des stations du massif des Alpes, IRSTEA, 2012. Françoise Miquel, Jacques Mougey et Georges Ribière, La Réhabilitation de l'immobilier de loisirs en France, Conseil général de l'environnement et du développement durable, Contrôle général économique et financier, 2010. 
Équipe Mit, Tourismes. 2, Moments de lieu, « Mappemonde », Belin, 2005.

Harvey L. Molotch, « The city as growth machine: Towards a political economy of place », American Journal of Sociology, vol. 82, n² 2, 1976, p. 309-332.

Jacques Mouriquand, L'Or blanc. Le système des sports d'hiver, Lieu commun, 1988.

Jacques Perret, Le Développement touristique local. Les stations de sports d'hiver, thèse de doctorat en économie du développement, université Pierre-Mendès-France de Grenoble, 1992.

Paul Pierson, « When effect becomes cause: Policy feedback and political change », World Politics, vol. 45, $\mathrm{n}^{\circ} 4,1993$, p. 595-628.

Michael J. Piore et Charles F. Sabel, The Second Industrial Divide: Possibilities for Prosperity, Basic Books, 1984.

Stanley C. Plog, "Why destinations area rise and fall in popularity ", Cornell Hotel and Restaurant Administration Quaterly, vol. 14, n 4, 1974, p. 55-58.

Michael E. Porter, Competitive Advantage: Creating and Sustaining Superior Performance, The Free Press, 1985.

Pierre Préau, « Le changement social dans une commune touristique de montagne : Saint-BonTarentaise (Savoie) ", Revue de géographie alpine, vol. 71, n 4, 1983, p. 407-429 [http:// www.persee.fr/doc/rga_0035-1121_1983_num_71_4_2546].

Pierre Préau, « Le changement social dans une commune touristique de montagne : Saint-BonTarentaise (Savoie) (deuxième article) ». Revue de géographie alpine, vol. 72, nº 2, 1984, p. 411-437 [ http://www.persee.fr/doc/rga_0035-1121_1984_num_72_2_2581].

François Servoin, La Commune de montagne, Economica, 1993.

Vincent Vlès, Service touristique local et Aménagement du territoire, L'Harmattan, 2001.

Marie Wozniak, L'Architecture dans l'aventure des sports d'hiver : stations de la Tarentaise, 1945-2000, Société savoisienne d'histoire et d'archéologie, 2006.

\section{NOTES}

1. Loi $n^{\circ}$ 85-30 du 9 janvier 1985 relative au développement et à la protection de la montagne. Les données de fréquentation fournies par Domaines skiables de France (2012) montrent en effet une relative stagnation des journées-skieurs depuis près de 10 ans.

2. Les cinq schémas interrégionaux de massifs de France métropolitaine évoquent la diversification des activités pour les stations de moyenne montagne, évocation laissant malgré tout place à une pluralité d'appropriations locales. Ainsi, deux grandes modalités de diversification s'observent: d'une part, une diversification focalisée sur la multiplication des types de prestations génériques importées puis adaptées au contexte montagnard, d'autre part, une diversification plutôt fondée sur le territoire et la spécificité locale. 


\section{RÉSUMÉS}

Cette contribution porte sur l'économie des sports d'hiver et entend questionner leur devenir. Ainsi, en relisant les travaux menés depuis quelques années relevant de différents champs disciplinaires, l'article se focalise sur la nature et l'intensité des trajectoires d'évolution des stations. Pour ce faire, l'analyse a caractérisé en leur sein la diversité des acteurs et de leurs logiques. En ce sens, les stations sont appréhendées comme des coordinations d'acteurs localisées et tout l'enjeu des recherches est d'évaluer le poids de certains facteurs, à l'image des stratégies plus ou moins territorialisées des opérateurs économiques ou bien encore l'impact des choix fonciers et immobiliers dans les destinations touristiques. L'influence de tels facteurs est à différencier selon le type de stations considérées, stations moyennes versus stations d'altitude. La compréhension de ces phénomènes peut ainsi contribuer à une action publique territorialisée et à son renouvellement.

This contribution concerns the economy of the winter sports and questions their future. So, by reading the works led since a few years (in various disciplinary fields), the article focuses on the nature and the intensity of the trajectories of evolution of ski resorts. In this sense, the analysis underlined the diversity of the actors and their logics in the ski resorts. The ski resorts are analyzed as located coordination's of actors and the stake is to assess the weight of different factors, like the strategies more or less territorialized of the economic operators either still the impact of the land use and real estate choices in the tourist destinations. The influence of such factors has to be differentiated according to the type of ski resorts (average ski resorts or high altitude ski resorts). The understanding of these phenomena can contribute to a territorialized public action and to its renewal.

\section{INDEX}

Mots-clés : stations de sports d'hiver, gouvernance, diversification touristique, immobilier de loisir, trajectoires d'évolution, aménagement du territoire

Keywords : ski resorts, governance, tourist diversification, real estate, trajectories of evolution, spatial planning

\section{AUTEURS}

\section{EMMANUELLE GEORGE-MARCELPOIL}

Université Grenoble Alpes, Irstea Grenoble, Développement des territoires montagnards - 2, rue de la Papeterie, BP 76, 38402 Saint-Martin-d'Hères - emmanuelle.george-marcelpoil@irstea.fr

\section{CORALIE ACHIN}

Université Grenoble Alpes, Irstea-DTM - 2, rue de la Papeterie, BP 76, 38402 Saint-Martin-d'Hères - coralie.achin@irstea.fr 


\section{GABRIEL FABLET}

Agence d'urbanisme de la région grenobloise, ex-doctorant Irstea-DTM, 21 rue Lesdiguières, 38000 Grenoble, gabriel.fablet@aurg.asso.fr

\section{HUGUES FRANÇOIS}

Université Grenoble Alpes, Irstea-DTM - 2, rue de la Papeterie, BP 76, 38402 Saint-Martin-d'Hères

- hugues.francois@irstea.fr 